\title{
Über das Optimieren
}

\section{Eberhard Wolff}

PD Dr. rer. soc., Redaktor Kultur, Geschichte, Gesellschaft

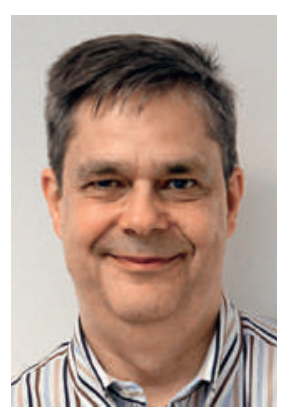

Steuern leicht gemacht lautete der Titel eines Ratgebers aus dem Beobachter-Verlag über zehn Neuauflagen hinweg. Jetzt kam der Steady-Seller überarbeitet als Der Steuerberater heraus - mit einem neuen Ziel im Untertitel, nämlich: «Steuern optimieren».

Haben Sie heute schon optimiert - oder es sich vorgenommen? Die Praxiserträge etwa, die Patientenbeziehungen oder die Parkplatzsuche? Das «Optimieren», oder zumindest das Sprechen darüber, geht um wie ein Gespenst. In der Alltagssprache, in der Werbung, auch in der Medizin. Achten Sie doch einmal darauf.

In diesem Blatt liest man ebenfalls immer häufiger vom Optimieren. Das digitale SÄZ-Archiv zeigt, dass der Wortstamm seit der Jahrtausendwende immer beliebter wurde. In der SÄZ des letzten Jahres ging es darum, Abläufe, Altersvorsorge, Befruchtungen, Behandlungsprozesse, Betreuung, Datennutzung, Effizienz, Hilfe, Kosten, Lebensstil, Leistungen, Medikation, Nutzen-Schaden-Verhältnisse, Performance, Prüfungen, Risikoabdeckung, Steuern, Studienmethoden, Versorgung, Weiterbildung, Zusammenarbeit und viele weitere Dinge zu optimieren oder optimal zu gestalten. Rekord: neun «Optimierungen» in einem Artikel. Für die Zukunft ist das aber sicher noch nicht das Optimum.

Je komplexer die Medizin wird, umso mehr müssen Ärztinnen und Ärzte beraten werden. So ehrenhaft und wichtig dieser Geschäftszweig ist, so sehr läuft er Gefahr, in die Formelhaftigkeit des Coaching-Jargons zu verfallen. Etwa: «Wir optimieren Ihre persönliche Performance!» Die Financial Times-Kolumnistin Lucy Kellaway hat sich Anfang Jahr über den Chef eines internationalen Beratungsunternehmens lustig gemacht, dessen Neujahrsmail an die Mitarbeitenden fast vollständig aus den leeren Phrasen der Beratersprache bestand [1]. Das O-Wort war nicht darunter, obwohl es zur klassischen Business- und CoachingRhetorik zählt. Denn das Optimale ist immer erwünscht und wird nie erreicht.

Eigentlich ist es ja schön, wenn Menschen etwas verbessern wollen. In mir wächst nur der Verdacht, dass Menschen das «Optimieren» oft dann floskelhaft einsetzen, wenn sie uns etwas verkaufen wollen. Das kann ein Produkt oder eine Dienstleistung sein, genauso aber auch eine Idee oder eine Rechtfertigung.

Auch Qualität ist etwas, das heute gerne "optimiert» wird. Der EMH-Verlag hat im letzten Jahr die nach- denklichen Briefe über Qualität in der (Hausarzt-) Medizin von Andrea Abraham und Bruno Kissling herausgebracht [2]. Darin finden wir bezeichnenderweise keinen "Optimierungs»-Jargon, allenfalls eine Kritik daran. "Optimieren» passt auf eine doppelte Art nicht in dieses Buch. Zum einen ist Qualität für Abraham und Kissling etwas Vielfältiges, Wandelbares; etwas, das aus dem Einzelfall jedes Mal individuell hergestellt werden muss. So etwas lässt sich kaum pauschal «optimieren".

Zum anderen kommt der Optimierungsjargon aus einer Denkwelt ökonomischer Kategorien, die gemeinsam mit dem Neoliberalismus aufblühte. Darin sind wir angehalten, in allen Lebenslagen «unternehmerisch" $\mathrm{zu}$ denken [3], auch wenn es nicht ums Geschäften geht. Dieses Phänomen ist im Gesundheitsbereich nicht unbekannt. «Optimieren» wird hier schnell auf die zahlenmässige Effizienzsteigerung reduziert. Auch dies passt nicht zu einem differenzierten Qualitätsbegriff.

«Selbstoptimierung» ist zurzeit auch ein Zauberwort der Fitness-Gesellschaft. Gleichzeitig ist «Selbstoptimierung» zu einer Art Schimpfwort derer geworden, die unsere Leistungs-, Ranking- und Evaluationsgesellschaft kritisieren. Diese Kritik fällt meines Erachtens aber zu oft auf die Floskel des Optimierens herein und nimmt das Reden über die Selbstoptimierung zu sehr für bare Münze. Beruhigend empfinde ich nämlich, dass die Wortführer des Optimierens diese Praktik in der Regel nur ziemlich suboptimal beherrschen. Wir erreichen gewöhnlich nicht das Beste, nicht einmal das Bestmögliche, und wollen es auch nicht unbedingt und immer - mit Ausnahme von ein paar Goldmedaillengewinnern vielleicht. Denn das pralle Leben ist der natürliche Feind jeder Optimierung. Deshalb sind wir auch nicht immer Unternehmer unserer selbst.

Bleiben Sie doch einfach dabei, Ihre Praxiserträge, Patientenbeziehungen oder Parkplatzsuche gut regeln zu wollen, statt sie zu optimieren. Das wäre am optimalsten.

\section{Literatur}

1 Kellaway L. Deloitte chief's new year memo is a classic in demotivation. Financial Times, 17. Januar 2016.

2 Abraham A; Kissling B: Qualität im der Medizin. Briefe zwischen einem Hausarzt und einer Ethnologin. Basel: EMH; 2015.

3 Bröckling U. Das unternehmerische Selbst. Frankfurt/M.: Suhrkamp; 2007. 